\title{
Holographic Weapons Sight as Crew Optical Alignment Sight
}

\author{
Nujoud Merancy* ${ }^{\mathrm{a}}$, Brian Dehmlow ${ }^{\mathrm{b}}$, Jack P. Brazzel ${ }^{\mathrm{c}}$ \\ ${ }^{\mathrm{a}}$ Booz Allen Hamilton, 2525 Bay Area Blvd, Houston, TX, USA 77058; \\ ${ }^{\mathrm{b}}$ L-3 Communications EOTech, 1201 E. Ellsworth Rd., Ann Arbor, MI, USA 48108; \\ ${ }^{\mathrm{c}}$ NASA, Johnson Space Center, 2101 NASA Parkway, Houston, TX, USA, 77058
}

\begin{abstract}
Crew Optical Alignment Sights (COAS) are used by spacecraft pilots to provide a visual reference to a target spacecraft for lateral relative position during rendezvous and docking operations. NASA's Orion vehicle, which is currently under development, has not included a COAS in favor of automated sensors, but the crew office has requested such a device be added for situational awareness and contingency support. The current Space Shuttle COAS was adopted from Apollo heritage, weighs several pounds, and is no longer available for procurement which would make re-use difficult. In response, a study was conducted to examine the possibility of converting a commercially available weapons sight to a COAS for the Orion spacecraft. The device used in this study was the XPS series Holographic Weapon Sight (HWS) procured from L-3 EOTech. This device was selected because the targeting reticule can subtend several degrees, and display a graphic pattern tailored to rendezvous and docking operations. Evaluations of the COAS were performed in both the Orion low-fidelity mockup and rendezvous simulations in the Reconfigurable Operational Cockpit (ROC) by crewmembers, rendezvous engineering experts, and flight controllers at Johnson Space Center. These evaluations determined that this unit's size and mounting options can support proper operation and that the reticule visual qualities are as good as or better than the current Space Shuttle COAS. The results positively indicate that the device could be used as a functional COAS and supports a low-cost technology conversion solution.
\end{abstract}

Keywords: holographic sight, human spaceflight, commercial space, spacecraft pilot, rendezvous, optical sensor, manual piloting

\section{INTRODUCTION}

NASA is currently developing a vehicle called the Orion Crew Exploration Vehicle to replace the Space Shuttle. This capsule shaped vehicle will primarily rely on relative navigation sensors and automation for rendezvous and docking; however, the Flight Crew Office at NASA has requested the inclusion of additional tools and techniques to provide situational awareness and support manual piloting in contingency situations. All piloted space vehicles since NASA's Gemini Program have carried a manual piloting aids such as a Crew Optical Alignment Sight (COAS). These tools have been used to support rendezvous, docking, and navigation in either primary or backup roles. A COAS is an optical device with fixed lateral and angular markings which provide measurement and targeting to external vehicles or points in space. An image of the view through the Orbiter COAS of Mir is shown in Figure 1. 


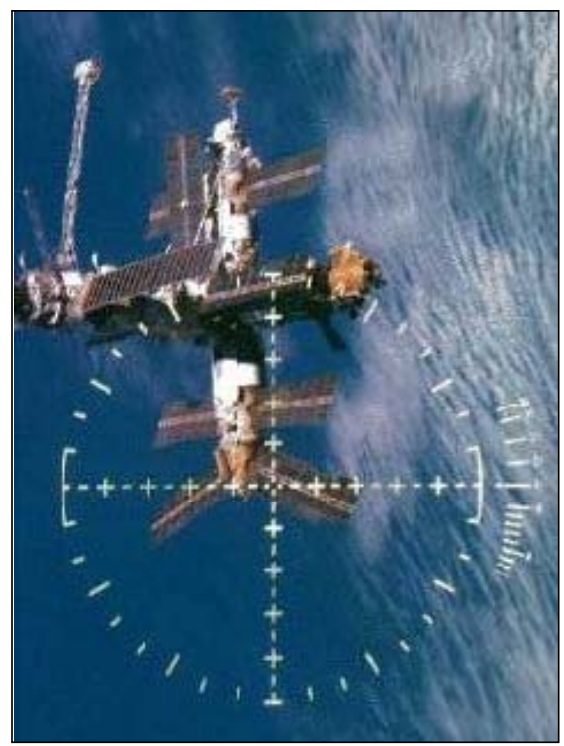

Figure 1. Orbiter COAS view of the Russian Mir Space Station.

\subsection{Orion Vehicle Integration}

In response to the crew request, investigation by the Orion Vehicle Integration Office concluded that integration of a COAS into Orion would be feasible. The Orion forward facing windows support a pilot's view of the docking axis and mounting of a unit would be possible in the existing vehicle design. . Volumetric and mass constraints would be a factor in the integration as with any hardware added to the manifest. Review of the current shuttle COAS, found it was adopted from Apollo heritage, weighs several pounds, and is no longer available for procurement and would, thus, be an unlikely candidate for use on any future vehicle. A survey was conducted of available hardware and commercially available weapons sights provided an attractive option for cost and schedule considerations. The ability to repurpose a weapons sight similar to those used by the military and law enforcement today would enable integration into Orion with a minimum impact to project resources. These self-contained sights also have physical qualities attractive to NASA in a COAS application including no external power requirements and are extremely lightweight.

\subsection{Study Scope}

Using a small scale grant program at NASA, a proposal was submitted to receive a small amount of funds to support investigation of the feasibility of converting a weapons sight to use as a COAS. The project was approved with a three month timetable allowed for completion of all activities. This activity was initiated with the objective of procuring a customized reflex sight and performing crew-in-the-loop simulations to evaluate its capabilities. Discussions were held between NASA Orion and L-3 EOTech engineering personnel to identify potential candidates and capabilities for implementation and ultimately produced a unit to apply in the evaluations.

Two evaluations were performed on the unit, the first was a form and fit evaluation performed in the Orion Low Fidelity Mockup located at the NASA Johnson Space Center to determine potential mounting locations for use. This evaluation involved two crewmembers and an off-the-shelf non-customized holographic weapon sight (EOTech XPS2-0) to determine appropriate positioning. The second evaluation was performed in the Reconfigurable Operational Cockpit (ROC) simulator which includes a mockup of the Orion cockpit with windows, vehicle displays, and flight controls and a wrap around projection of the external environment. This manual piloting evaluation allowed the crewmembers to fly the Orion vehicle using a customized sight COAS in an ISS rendezvous simulation. The customized sight was a standard XPS sight fitted with a modified COAS holographic reticule. These evaluations utilized experienced flight crew pilots and rendezvous engineering and operations experts for support. In all evaluations, qualitative comments were received to judge the device performance and capabilities. 


\section{CREW OPTICAL ALIGNMENT SIGHT FUNCTION}

\subsection{Mechanics of Operation}

The COAS for the Space Shuttle Orbiter consists of a lamp with an intensity control, a reticule, a barrel-shaped housing, a mount, a combiner assembly and a power cable [1]. The reticule consists of a 10-degree circle (i.e., 5-degree radius), vertical and horizontal cross hairs with 1-degree marks, and an elevation scale on the right side of the circle from minus 10 degrees to 31.5 degrees (see Figure 1). The reticule is projected on a combining glass focused on infinity. The COAS is 9.5 by 6.0 by 4.3 inches, weighs 2.5 pounds, and requires 115 -volt ac power for reticule illumination and is diagramed in Figure 2.

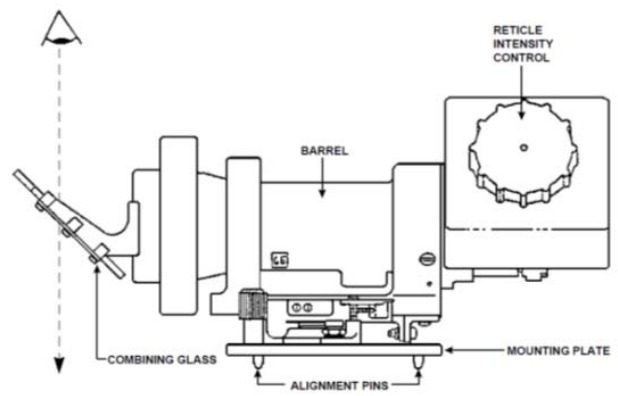

Figure 2. Orbiter COAS Side View Diagram

The COAS, pictured in Figure 3, on the Orbiter serves a variety of functions during ascent, on-orbit, and deorbit/entry phases of flight. The COAS is mounted in the forward window looking out over the nose (see Figure 4) to allow the crew to monitor the vehicle's attitude orientation during ascent and deorbit thrusting periods. The COAS is also used to check alignment of the payload bay doors during closure prior to deorbit by mounting it in the aft window looking into the payload bay. During on-orbit operations, the COAS is mounted in the overhead window looking out the top of the cockpit to provide a backup to the star trackers for inertial measurement unit (IMU) alignment and to allow the crew to visually monitor the approach to a target vehicle for rendezvous and docking missions.

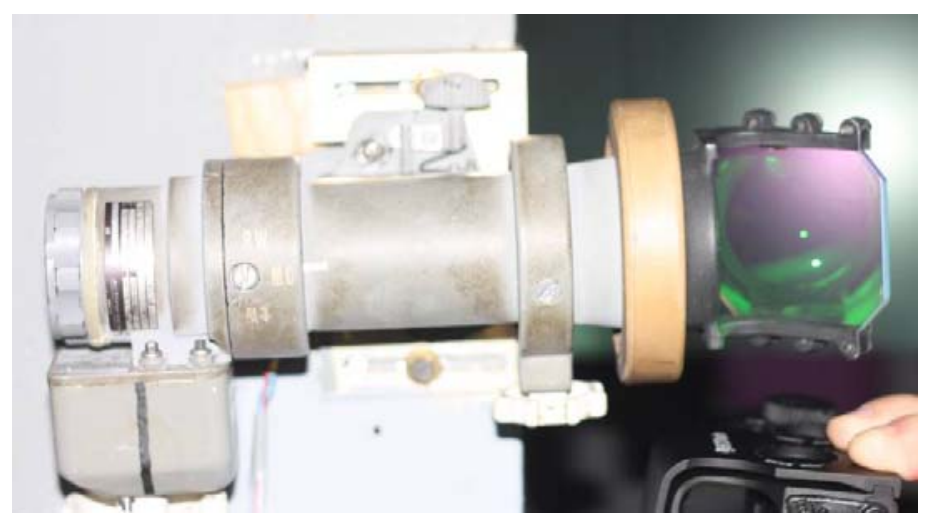

Figure 3. Orbiter COAS 


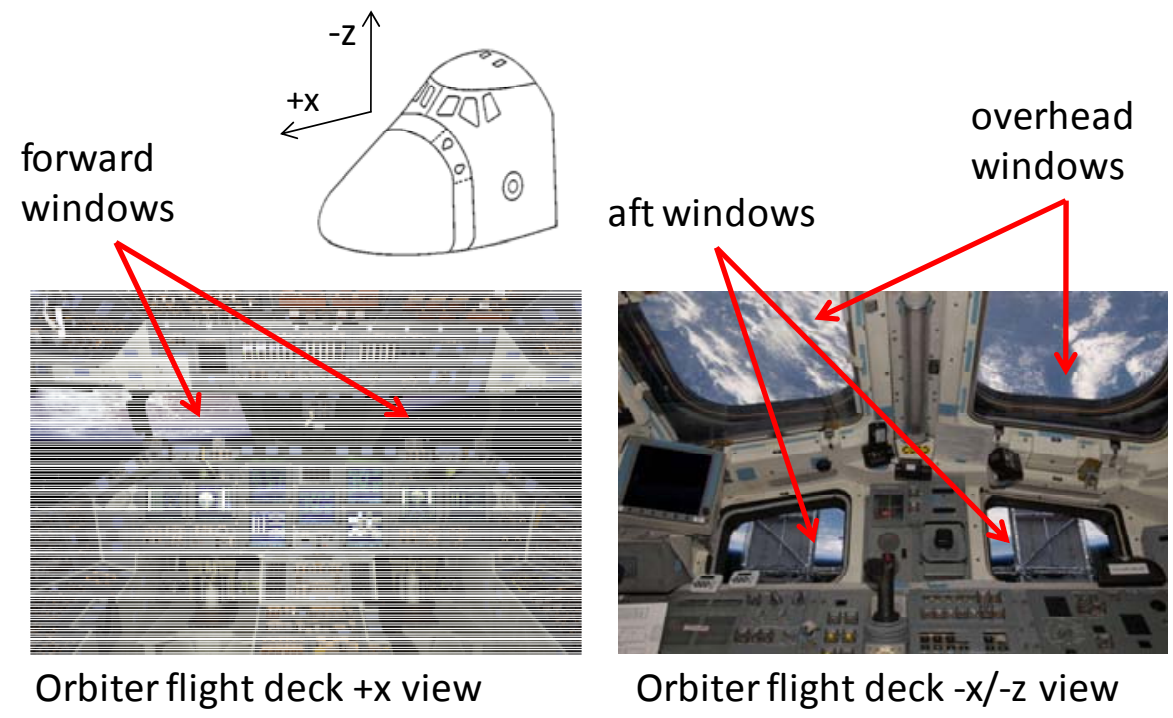

Figure 4. Orbiter Flight Deck Windows

The crew uses the COAS to align IMUs under the following conditions 1) when the IMU misalignment is greater than the star trackers can handle, 2) when the star trackers have failed to verify the correct star or the star tracker is not available because it is being used to track a target vehicle during rendezvous, and 3) to improve the relative Orbiter-totarget state vector during the rendezvous phase. The crew at the aft flight deck station manually maneuvers the Orbiter while looking out the COAS in the right overhead window until the selected star is in the field of view. The crew continues maneuvering the Orbiter until the star crosses the center of the reticule. At the instant of crossing, the crew takes a mark by depressing the attitude reference push button in the cockpit. At the time of the mark, the onboard software stores the gimbal angles of the three IMUs. Good marks for two stars are required for a successful IMU alignment. By knowing the identification of the star being sighted and the COAS mounting location on the Orbiter, the onboard software determines a line-of-sight vector from the COAS to the star in an inertial coordinate system. Line-ofsight vectors to two stars define the attitude of the Orbiter in inertial space. This attitude can be compared to the attitude defined by the IMUs, and if the IMUs are in error, they can be realigned to the more correct orientation by the COAS sightings.

During a rendezvous mission, as the Orbiter approaches the target vehicle, the crew may also use the COAS to obtain the range and line-of-sight angle to the target, as well as obtaining a qualitative idea of range rate and target normal line-ofsight rate. The crew manually flies the Orbiter along an approach trajectory toward the target vehicle by making inputs to the translational hand controller (THC) in the desired direction. The COAS is used to aid the pilot in determining the direction and number of THC inputs to control the trajectory to the target vehicle. The COAS allows the pilot to visually see and quantify lateral motion, which is essential in determining the appropriate manual THC inputs to control the trajectory. This phase of flight occurs from about $2000 \mathrm{ft}$ to the final docking or capture.

\subsection{Updated Reticule Pattern}

During execution of the reticule customization task during the study, NASA rendezvous engineers noted that the circular Orbiter reticule pattern presented limitations in the pilot's ability to use the COAS. Specifically, the reticule pattern limits the ability of the flight crew to estimate position when off axis in both vertical and horizontal directions, or the "corners" of the field of view. To remedy this situation, the pattern was improved to provide a square reference field of view marking. This will improve the crew's ability to quantify lateral target motion when a target is dispersed outside the 10-degree circle. Figure 5 shows a comparison of the circular Orbiter and square Orion COAS pattern drafted for this study. 

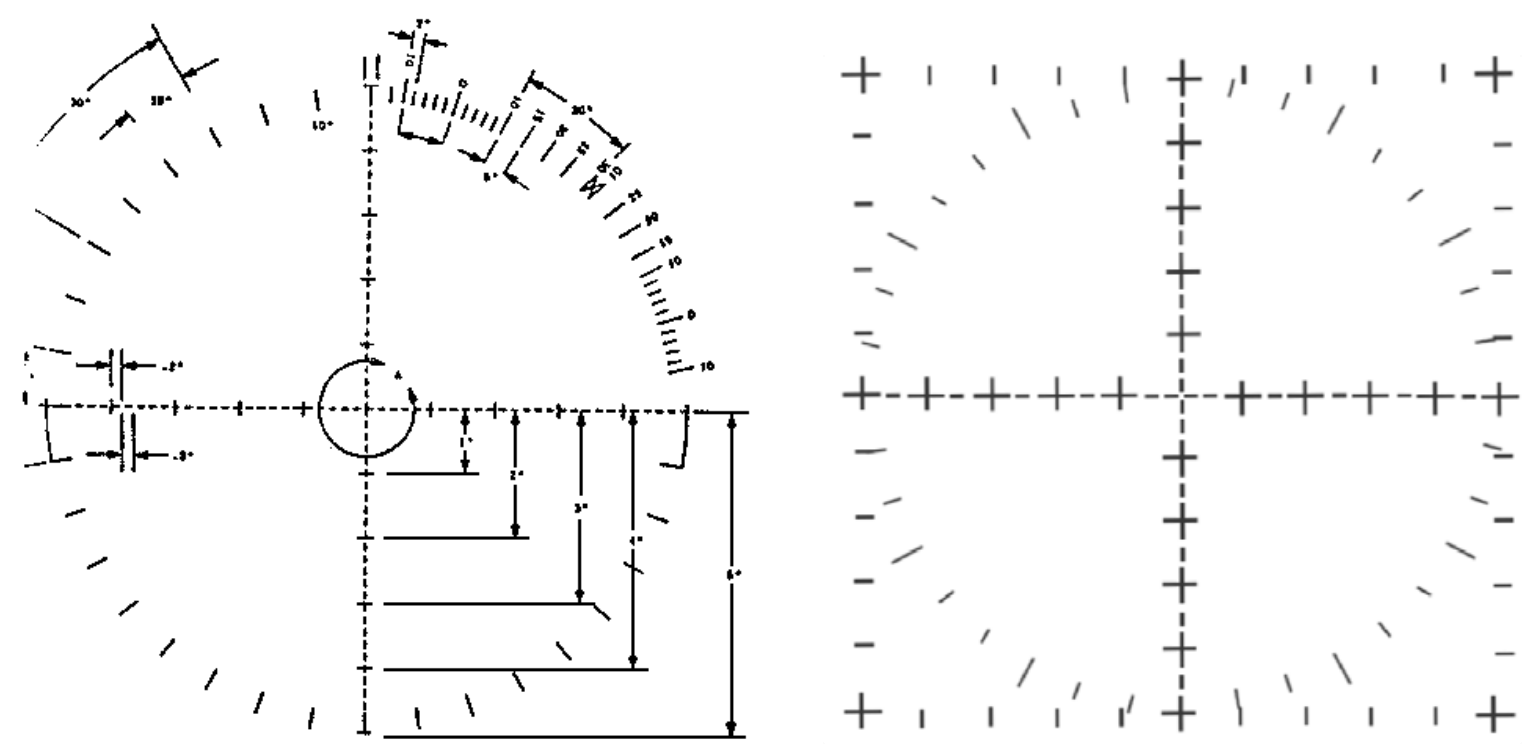

Figure 5. Orbiter (left) and Orion (right) COAS Reticules

\section{SIGHT TECHNICAL DETAILS AND FUNCTION}

\subsection{Mechanics of Operation}

The Holographic Weapons Sight (HWS) [2][3] utilizes a pre-recorded hologram to display a virtual image of the desired reticule pattern. The hologram image appears as a monochromatic red $(650 \mathrm{~nm})$ pattern projected at a large distance (typically 300 meters) in front of the sight. The image is free of parallax error at this distance, and exhibits very low parallax ( $<1-2$ arc-minutes) at other distances beyond 100 meters.

The size of the reticule is a fixed subtended angle, which is determined during the recording process. The desired reticule pattern is first created as a photographic mask whose size is matched to the desired subtended angle of the virtual image. The pattern is then recorded into a photographic emulsion using the holographic recording set-up shown in Figure 6. After chemical processing, the result is a volume transmission hologram with high transmittance and low scatter. The virtual image of the reticule pattern is generated when the hologram is illuminated by a laser beam, as illustrated in Figure 7. The illuminating laser beam size, wavelength, angle of incidence, and degree of collimation must match the recording conditions. Because the image hologram lacks any optical power, the view through the holographic sight is not magnified (i.e. unity or $1 \mathrm{X}$ magnification). 


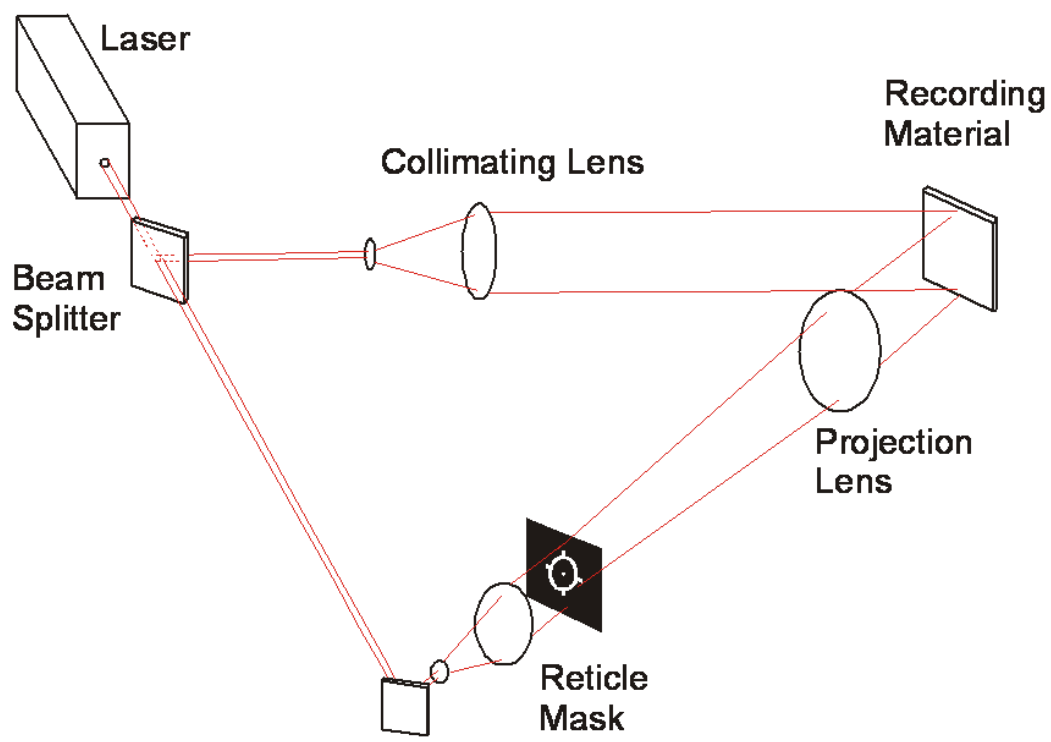

Figure 6. Holographic Recording Set-up

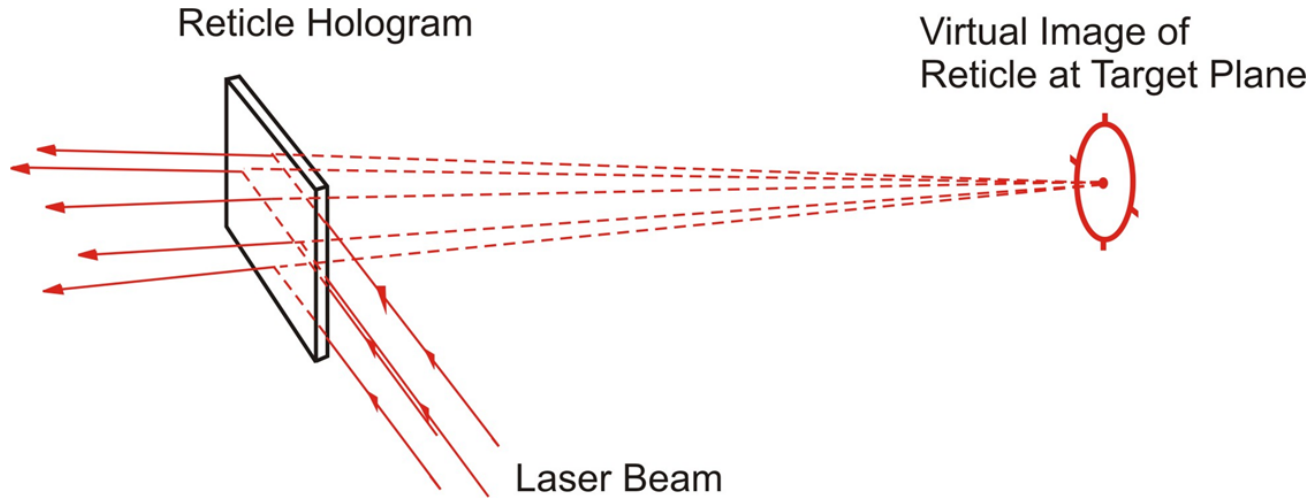

Figure 7. Holographic Image Reconstruction

\subsection{Sight Reticule Adaptation}

Hologram-based reticules offer several advantages over other non-holographic reflex sights for the COAS application. First, the hologram maintains a parallax-free image over the entire viewing aperture, so there is no need to center the reticule in the aperture. This permits faster visual capture of the target, and flexibility in the viewer's position. Second, the high transmittance and unity magnification allow the user to keep both eyes open and focused on the target. This allows normal binocular depth cues to remain intact. Unlike classical optical telescopes, holographic sights do not form an exit pupil. This allows viewing distance and position to vary without dimming or obscuring the image.

The most significant advantage of holographic sights is the ability to make large and unique reticule patterns. Holographic sights can change reticule patterns simply by changing the mask used during the recording process. Despite this flexibility in size and shape, limitations still exist. In particular, the sight's viewing aperture combines with the viewing distance (eye to HWS) to define the largest reticule that can be viewed simultaneously. The sight's viewing aperture is roughly $30 \mathrm{~mm}$ wide by $20 \mathrm{~mm}$ high, which creates a field-of-view (FOV) of 5.7 by 3.8 degrees at a viewing distance of 0.3 meter (approximately 12 inches). Holographic sights for consumer, military, and law enforcement purposes routinely use a 1.08 degree (65 arc-minute) circle with a central 1 arc-minute dot. This pattern size fits 
comfortably within the existing HWS FOV, but similar considerations placed an upper limit on the useful COAS reticule size.

The desired Orion COAS reticule shown earlier is a 10-degree square with $1^{\circ}$ pitch/yaw axis increments and $10^{\circ}$ roll axis increments. This pattern is too large and sparse to use effectively with the available HWS FOV. A comparison between the sight FOV and COAS reticule is shown in Figure 8. In addition, this large FOV requires a fundamentally different mask technology. As a result, a smaller 4-degree square was chosen to demonstrate holographic COAS feasibility. This pattern was able to maintain the desired pitch, yaw, and roll axis increments. A comparison of the various reticule patterns is shown in Figure 9. While this smaller pattern remains slightly larger than the standard FOV, all portions of reticule can be seen if the crew member's head is perturbed on any axis or closer, which is easily induced in a restraint free environment. Because the sight is parallax-free, such shifts do not move the pattern's center relative to the target spacecraft.

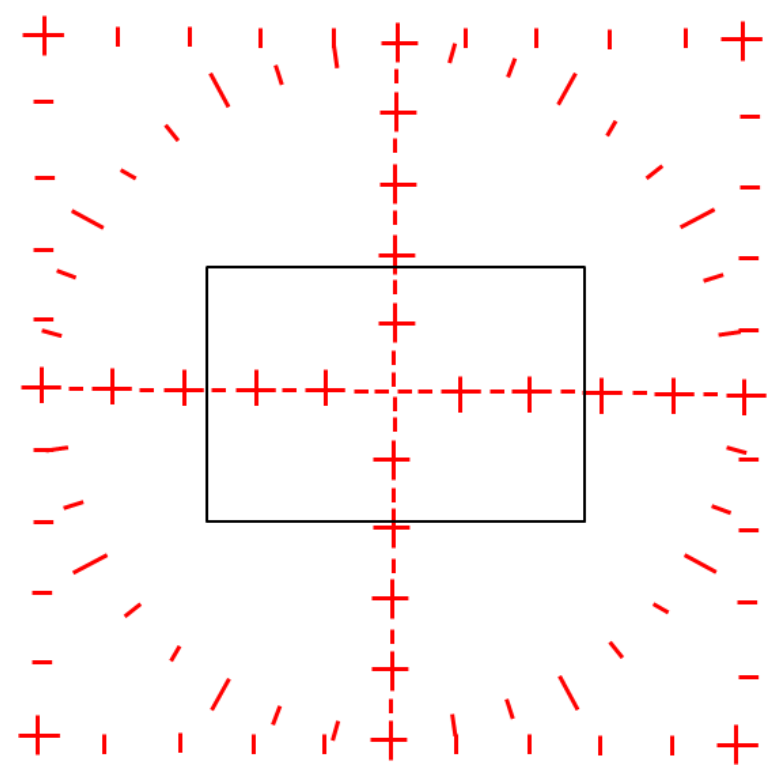

Figure 8. HWS FOV relative to 10 degree Orion Reticule 


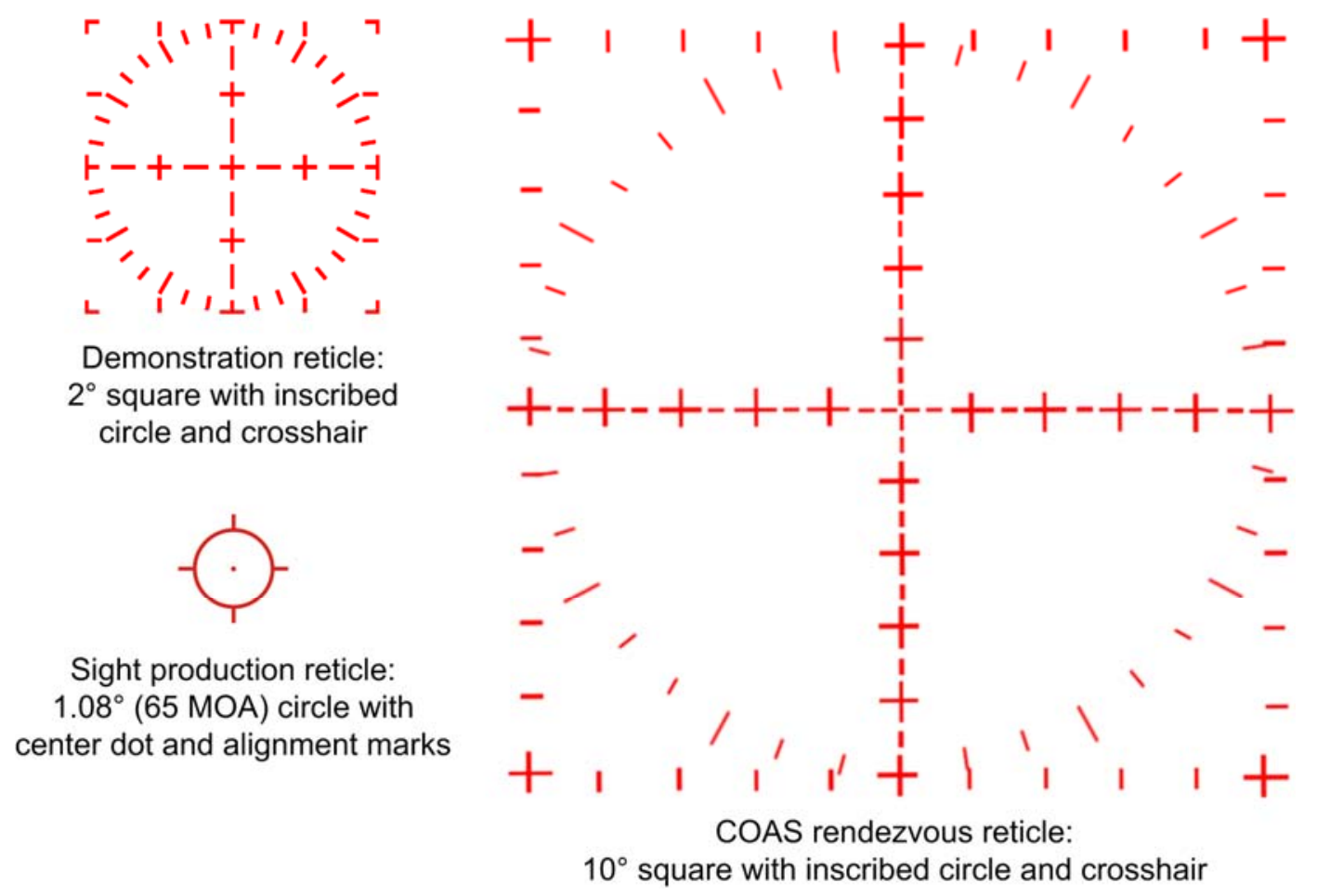

Figure 9. Relative Sizes of Different Reticules

\section{EVALUATIONS AND RESULTS}

\subsection{Facilities}

Two Orion facilities were utilized to support evaluations of the updated COAS unit. The initial evaluation with the COAS was to determine the fit and potential mounting locations for the pilot's view in the Orion low fidelity mockup at the Johnson Space Center. The mockup is built to scale for both external and internal dimensions and includes the current cockpit arrangement. This facility includes windows, displays and controls, and the seat configuration necessary to determine if the COAS unit could be integrated in a suitable orientation and location to support its operation. In particular, this facility provides the inner mold line (cabin walls) of the pressure vessel relative to the pilot position for window viewing to support the form and fit evaluation.

The second facility utilized was the Reconfigurable Operational Cockpit (ROC) to evaluate the operation of the device. The ROC also provides a rapid proto-type layout of the Orion cockpit also with the windows, displays, and controls in their proper locations. This facility includes a wrap around screen to support external viewing of the projected scene from a six degree of freedom simulation. The displays and controls are operational and integrated into the simulation to allow real-time pilot interaction.

\subsection{Form and Fit Evaluation}

The COAS evaluation in the mockup was performed with the support of flight crew members, RPOD engineers, rendezvous flight controllers, and a representative for the mockup. This evaluation was performed using an unmodified, loaner XPS unit from L-3 EOTech. This allowed for an initial determination on the size and fit of the device while the customized unit was in development. Feedback received during the evaluation is detailed below and an image is shown in Figure 10.

- Mount away from the window frame with at least 10deg of viewing above and below COAS visible.

- Vertical, downward direction of viewing is most important.

- Suggested sideways mount from the right center of the frame would be most desirable. 
- Regarding the COAS hardware a crisp, bright pattern is ideal and was delivered in the sight.

- Variable brightness is a definite plus.

- Overall small outer dimensions of the COAS were well liked.

- A sturdy mounting bracket to facilitate ground calibration would be ideal.

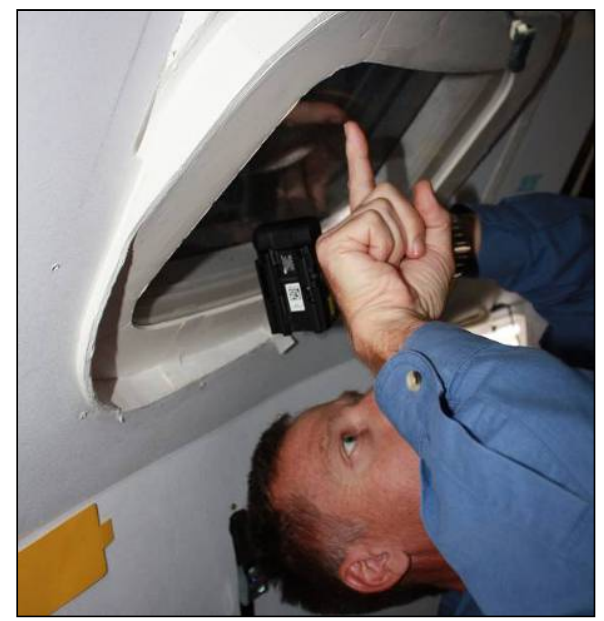

Figure 10. Flight crewmember indicating ideal mounting location in Orion Low Fidelity Mockup

Using the feedback received in the mockup evaluation, a mount was created using rapid prototyping parts for the standup half-dome Orion simulator in the ROC. This mount places the COAS in the location identified by the crew and would be representative of placement in a fully developed Orion vehicle. Figure 11 shows the COAS mounted in the ROC prior to the simulations. This rapid-prototype mount is not intended to represent a flight system as it does not provide calibration or repeatability and is significantly larger and heavier than what would be necessary on-orbit.
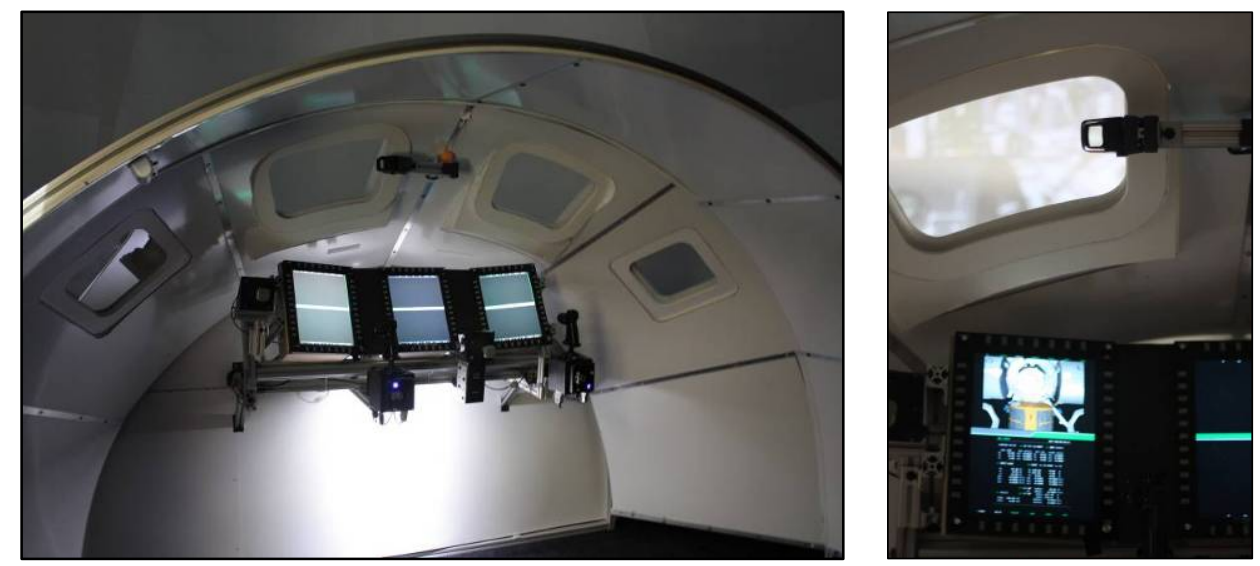

Figure 11. Weapons Sight COAS mounted in Orion Stand-up Simulator in the ROC

\subsection{Manual Piloting Evaluation}

Evaluation of the COAS was performed using a real-time pilot-in-the-loop simulation to fly an Orion approach and docking to ISS. This evaluation was conducted with three crew members, two of which also supported the prior mockup evaluation, and the same engineering and operations personnel from the fit check. The reticule customized unit used to support this evaluation effectively demonstrates the ability to implement this commercial device as a COAS. All of the personnel present commented positively on the COAS unit and feel that this unit would provide an excellent hardware solution. One of the crew members called it "perfect" and an option the crew would love to have available regardless of the vehicles they are asked to fly in the future. Evaluation results from the simulation are listed below and Figure 12 illustrates the pilot's perspective in the simulation. 
- Device satisfied the general needs and desires of a COAS very well.

- One evaluator stated that the reticule was better/crisper than the Orbiter COAS's bulb illumination.

- The mounting location was acceptable and provided the desired amount of vertical field of view above and below the COAS through the forward facing Orion window.

- The left side of the Orion window has a greater downward viewing angle available; however, placing the COAS there felt "unnatural" and would force the pilot out of the nominal position.

- Initial thoughts that the protective "cage" around the window would be a visual interference were unfounded and did not affect viewing.

- The new COAS reticule pattern was considered a positive change; however, the +/-5deg FOV pattern is highly desired to support the available trajectory space.

- Increase of the COAS outer dimensions to support a larger FOV would not be problematic.
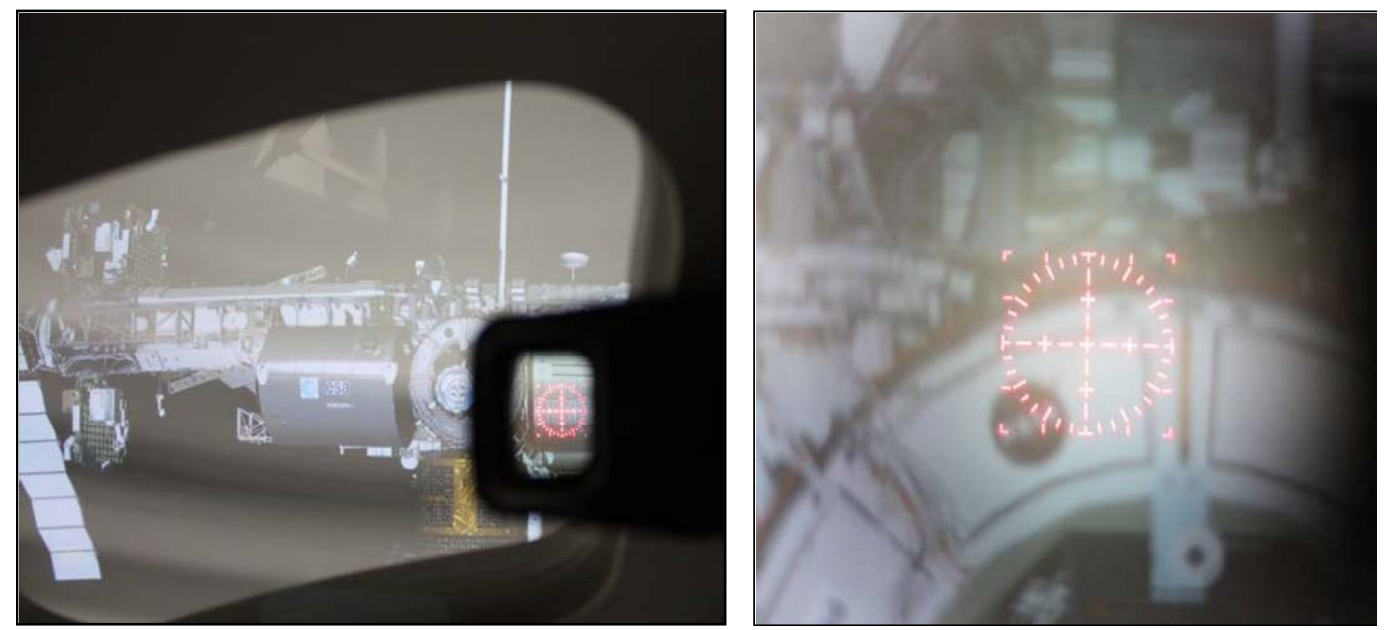

Figure 12. Pilot's eye view through COAS in ISS rendezvous simulation

A positive anecdotal result of the evaluation involved the ROC screen projections. Initial mounting of the COAS in the ROC was "eyeballed" parallel to the floor which should be aligned to the vehicle positive x-axis. However, during the Orion far-field rendezvous it was noted that the COAS was not pointed directly at the ISS as was expected. To align the COAS, the unit had to be adjusted to approximately a ten degree down angle. Conversations with ROC support personnel identified that the simulation window projections were in fact uncalibrated and had a known downward shift due to limitations of the physical space. Without an available visual reference source such as a COAS, projection calibration has not been required or previously discernable to pilots. As a result, use of the COAS to recognize this offset indicates the positive contribution such a device has to manual piloting.

\section{FUTURE CAPABILITIES}

\subsection{Sight Hardware Opportunities}

Accommodating the full 10-degree COAS reticule pattern requires a sight approximately 2-3 times larger than the current version. Since the time of this study, a larger MK56 sight has been developed for military uses. This sight is 1.75 times larger than the standard sight, as shown in Figure 13. This new sight size creates a FOV of approximately 10 by 6.7 degrees at a viewing distance of 0.3 meter. If a sight of this size is used as the basis for COAS, the full reticule size is viewable at a distance of $200 \mathrm{~mm}$ or less which can be easily accommodated by flight crew positioning. Since this sight is already being developed for other applications, space deployment might be accomplished with significantly lower cost than developing a totally new hardware platform. Unlike the XPS, the MK56 does not include a protective hood (cage). This positively addresses one of the users' concerns about outward visibility obstruction. 


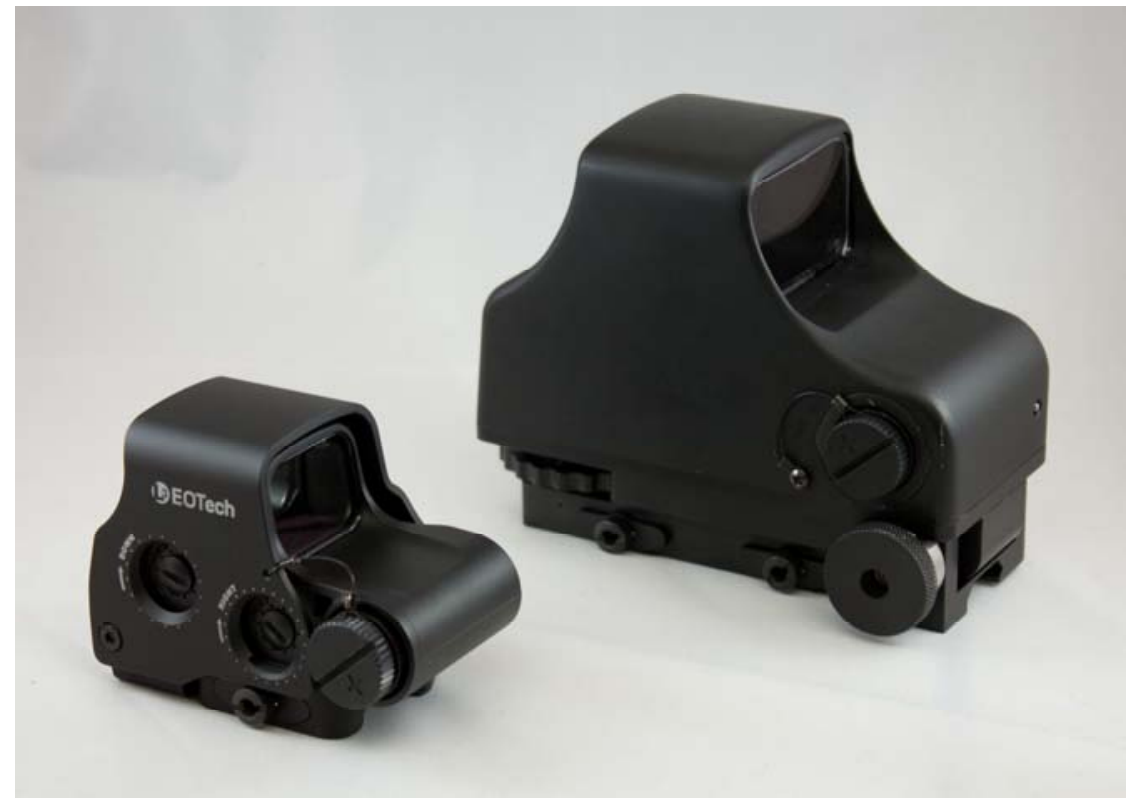

Figure 13. Size Comparison between Standard (XPS) and Larger (MK56) Sight Platforms

As noted earlier, the 10-degree FOV also requires development of new mask technology. The projection lens in the holographic recording set-up creates a sharp off-axis image only when the mask falls on a spherical surface. Restricting the mask to a planar surface introduces focus errors near the edges of the image. These errors can be ignored with a small FOV, but become progressively more significant as the FOV expands. While current technology allows only planar or cylindrical masks, it might be possible to fabricate a spherical mask. Further investigation is needed to determine the feasibility of spherical mask construction, as well as to determine if the level of performance would be acceptable.

\subsection{Spacecraft Opportunities}

While this device was studied with the intent for future inclusion on the NASA Orion spacecraft, the use of a weapons sight as a COAS would be applicable to any piloted vehicle. This low cost solution would be beneficial to any crewed spacecraft whether it were government or commercially funded. The COAS integration into a vehicle could be conducted to support several uses including rendezvous, docking/undocking, navigation alignment, and serve as a contingency device in the event of sensor or automation failures. Human spaceflight is a growing industry and pilotability will be a concern for any vehicle.

\section{CONCLUSION}

Crew Optical Alignment Sights continue to be a key component for the situational awareness of piloted spacecraft. These or similar devices have been successfully utilized on all U.S. piloted spacecraft with requirements for relative positioning to a target such as rendezvous or docking. This study examined the ability to modify the reticule of a commercially available weapons sight and apply it as a COAS utilizing both the Orion Low Fidelity Mockup and realistic rendezvous simulations in the ROC. The L-3 EOTech holographic weapons sight was selected and successfully demonstrated to meet the user needs and qualitative requirements for such an application. Limitations of the size of the field of view were accepted in this study to allow for an available demonstration unit to be assessed. However, a weapons sight under development by L-3 EOTech has already been identified with capabilities to support the full field of view required by a NASA COAS. This study has identified an exceptional candidate in the weapons sight for application as a COAS to support human space exploration in the $21^{\text {st }}$ Century. 


\section{REFERENCES}

[1] Sarafin, Michael, “Guidance, Navigation, and Control Section 18. Crew Optical Alignment Sight”, NASA Shuttle Operations Handbook, JSC-18863 (1998).

[2] Tai, Anthony M., et.al. “Compact Holographic Sight”, US Patent 5483362, 9 January 1996.

[3] Tai, Anthony M., et.al. “Lightweight Holographic Sight”, US Patent 6490060, 3 December 2002.

Notice: This publication consists of L-3 Communications Corporation general capabilities information that does not contain controlled technical data as defined within the International Traffic in Arms (ITAR) Part 120.10 or Export Administration Regulations (EAR) Part 734.7-11. 